\title{
Cuidado farmacêtico domiciliar na Estratégia Saúde da Família
}

\author{
I ${ }^{1}$ Jonas Bastos Santos, 2 Thais Mendes Luquetti, ${ }^{3}$ Selma Rodrigues Castilho, \\ ${ }^{4}$ Sabrina Calil-Elias I
}

Resumo: Este trabalho desenvolveu e avaliou uma ferramenta para auxiliar no planejamento, execução, registro de dados e avaliação das ações da visita domiciliar farmacêutica na equipe de Estratégia Saúde da Família. A primeira fase consistiu na construção da ferramenta de visita domiciliar farmacêutica, através de grupo focal e análise de discurso. Discutiu-se a visão dos profissionais sobre o papel do farmacêutico e sua prática profissional, em especial na visita domiciliar em unidades de atenção primária à saúde. Na segunda fase, utilizou-se o formulário desenvolvido na etapa anterior durante a visita domiciliar por 32 farmacêuticos, e avaliaram-se adequação, clareza, efetividade/precisão, exequibilidade e sugestóes para a ferramenta. Todos os participantes do estudo efetivamente realizam visita domiciliar na Estratégia de Saúde da Família no município do Rio de Janeiro. Segundo avaliação expressiva dos participantes, o instrumento foi considerado adequado, claro, efetivo, preciso e exequível para utilização na prática de visita domiciliar; representando importante auxílio ao profissional na realização do cuidado farmacêutico domiciliar naquele município.

> Palavras-chave: Atenção Primária à Saúde; cuidado farmacêutico; visita domiciliar.

\author{
1 Faculdade de Farmácia, \\ Universidade Federal Fluminense. \\ Niterói-RJ, Brasil (jonasbastos. \\ uff@hotmail.com). \\ ORCID: 0000-0001-9178-6499 \\ ${ }^{2}$ Faculdade de Farmácia, \\ Universidade Federal Fluminense \\ Niterói-RJ, Brasil (thaisImendes@ \\ gmail.com). \\ ORCID: 0000-0001-8739-8733 \\ ${ }^{3}$ Faculdade de Farmácia, \\ Universidade Federal Fluminense. \\ Niterói-RJ, Brasil (selmarc@ \\ id.uff.br). \\ ORCID: 0000-0003-0272-4777 \\ ${ }^{4}$ Faculdade de Farmácia, \\ Universidade Federal Fluminense. \\ Niterói-RJ, Brasil (sabrinacalil@ \\ id.uff.br). \\ ORCID: 0000-0002-2026-1510
}

Recebido em: 20/04/2019 Aprovado em: 03/03/2020 Revisado em: 30/06/2020 


\section{Introdução}

As açôes da Estratégia Saúde da Família (ESF) são complexas e precisam dar conta das necessidades de saúde da população, em nível individual e/ou coletivo, influindo na saúde e na autonomia das pessoas e nos determinantes e condicionantes de saúde da comunidade (FIGUEIREDO, 2017). Para isso, é necessário trabalhar de forma compartilhada, ampliando a integração dos profissionais de saúde no trabalho conjunto que busque a integralidade da atenção à saúde (CENTA; ALMEIDA 2003; ALMEIDA; MISHIMA, 2001).

$\mathrm{O}$ trabalho em equipe é considerado um dos pilares para aumentar a capacidade de análise e intervenção sobre os problemas e demandas de saúde, tanto em aspectos clínicos quanto sanitários (ALMEIDA; MISHIMA, 2001; OLIVEIRA; SPIRI, 2006) A participação efetiva e integral do farmacêutico passa a ser fundamental na ESF, seja através do Núcleo de Apoio à Saúde da Família (NASF) ou diretamente na unidade de saúde, proporcionando o desenvolvimento de atividades relacionadas com o trabalho em equipe e com o usuário (PEREIRA et al., 2005).

Visando à produção de novos modos de cuidado, a ESF propóe a visita domiciliar (VD) como instrumento central no processo de trabalho das equipes (BORGES; SANTOS, 2011). A VD é destinada principalmente ao usuário que possui dificuldade que o impede de se deslocar até o serviço de saúde. É a partir da visita da equipe no domicílio que são traçados o plano e as estratégias de açôes da equipe junto à família, uma atividade que se constrói fora do espaço hospitalar e dos ambulatórios de especialidades, com características desenhadas e voltadas a prevenção de doenças e promoção da saúde (BRASIL, 2003). Ainda segundo Santos e Moraes (2011), a partir dessa prática é possível avaliar as condições de habitação, saneamento, aplicar medidas de controle das doenças transmissíveis e parasitárias, promover orientaçôes para o autocuidado das doenças crônicas não transmissíveis e desenvolver outras açôes de educação em saúde.

Para sua efetividade são necessários vários procedimentos, entre eles priorizar os grupos mais vulneráveis (idade avançada, doenças crônico-degenerativas, dependência física ou psíquica, situaçôes terminais e Aids), definir os objetivos da VD, reunir todas as informações possíveis sobre a família e o paciente (prontuários, informaçôes dos agentes comunitários de saúde), reunir materiais apropriados para o atendimento, dentre outros (BRASIL, 2012). Outro fator importante para a assistência domiciliar 
ser bem-sucedida é o cuidador, pois sem ele não há continuidade da atenção. $\mathrm{O}$ cuidador pode ser membro da família ou da comunidade que, idealmente, deve atuar como colaborador para a equipe de saúde e a família, pois em geral não tem vínculo legal com a instituição que presta assistência domiciliar (SAVASSI; DIAS, 2006).

Para impactar nos diversos fatores que interferem no processo saúde-doença, é importante que a VD seja realizada por equipe multiprofissional e com prática interdisciplinar. Desta forma, terá maior probabilidade de atender a todas as orientações e intervençôes necessárias para melhoria da qualidade de vida do paciente. ${ }^{9}$

As atividades farmacêuticas no contexto do cuidado domiciliar são regidas pela Resolução no 386/2002, do Conselho Federal de Farmácia. Pode-se citar como principais atribuiçôes do farmacêutico no cuidado domiciliar orientaçóes quanto: ao uso e efeitos adversos dos medicamentos; as interaçóes medicamentosas; as vias de administração dos medicamentos, como por exemplo, por via de sondas parenterais; ao armazenamento e descarte de medicamentos da forma correta, garantindo o uso racional dos medicamentos e melhorando a segurança do paciente (BRASIL, 2017).

O processo de cuidado ao usuário, desenvolvido pelo farmacêutico, compóese, em sua grande maioria, de quatro etapas: a coleta e organização dos dados do usuário; avaliação e identificação de problemas relacionados à farmacoterapia; pactuação de um plano de cuidado com o usuário; e seguimento individual do usuário, quando necessário (CORRER et al., 2011; BRASIL, 2015). Desse modo, todas as açôes construídas com o paciente na unidade de saúde ou no domicílio devem ser registradas, incluindo a educação em saúde, encaminhamento a outros profissionais da saúde, intervençôes na farmacoterapia, medidas não farmacológicas como incentivo à atividade física, a reeducação alimentar entre outros (CARDOSO et al., 2003). O objetivo deste trabalho visa desenvolver um instrumento de visita domiciliar farmacêutica através da experiência desses profissionais e posteriormente avaliar o instrumento com a aplicação durante a rotina desse profissional.

\section{Métodos}

Trata-se de estudo quanti-qualitativo, descritivo e analítico. O estudo foi realizado com farmacêuticos da Estratégia de Saúde da Família do município do Rio de Janeiro e foi dividido em duas etapas. A primeira etapa teve como objetivo a construção da ferramenta para o cuidado farmacêutico na VD. A segunda etapa 
consistiu na aplicação desta ferramenta pelos farmacêuticos que executam a VD e posterior avaliação do instrumento por estes profissionais.

O presente trabalho foi aprovado pelo Comitê de Ética em Pesquisa (CEP) do Hospital Universitário Antônio Pedro e da Secretaria Municipal de Saúde do Município do Rio de Janeiro, através do CAAE 59722516.1.0000.5243 (Parecer número 1.808.822).

\section{Primeira etapa}

Foram convidados 10 farmacêuticos para o desenvolvimento do constructo, com experiência mínima de um ano em VD. Utilizou-se a técnica do grupo focal para explorar as percepçóes dos farmacêuticos que atuam em unidades de atenção primária à saúde sobre seu papel e sua prática profissional. A técnica do grupo focal foi escolhida, pois permite que os participantes dialoguem, a partir da discussão com foco em tópicos específicos e diretivos, emergindo assim, diferentes pontos de vista (KIND, 2004; RESSEL, 2008). Como procedimento de coleta de dados, permite ao pesquisador ouvir vários indivíduos ao mesmo tempo, obter várias informações, sentimentos e experiências acerca de determinado tema (SILVA; ASSIS, 2010). Assim, as atitudes e percepçôes dos participantes não são desenvolvidas isoladamente, mas através da interação entre eles.

Para discussão no grupo focal, foi inicialmente desenvolvido um formulário de visita domiciliar farmacêutica, construído com base nos estudos encontrados na literatura. Utilizaram-se trabalhos que identificam conceitos e noçóes a respeito da atenção primária, da assistência farmacêutica, assim como o papel do farmacêutico no contexto da APS (SILVA et al., 2016).

O formulário de visita domiciliar farmacêutica foi dividido em blocos, para melhor orientação da discussão, a saber: Perfil do paciente; História social; Farmacoterapia atual; Acesso aos medicamentos; Problemas de saúde / Queixas; Adesão ao tratamento; Terapias alternativas/complementares; Identificação de problemas relacionados a medicamentos (PRM); Intervençôes farmacêuticas; Finalização, agendamento e outras ações pactuadas com o paciente.

Para a análise do formulário, utilizou-se o índice de validade de conteúdo (IVC). O IVC mede a proporção ou porcentagem de juízes que estão em concordância sobre determinados aspectos do instrumento e de seus itens. Assim, o resultado do painel de experts (juízes) é usado para definir os itens que serão mantidos, revisados 
ou eliminados (SILVA; ASSIS, 2010). Uma vez que o índice de concordância é a medida mais utilizada para determinar a validade de conteúdo, o índice de, no mínimo, $80 \%$ de concordância entre os avaliadores refere-se à boa qualidade dos itens. Após a análise, foram levantadas, para cada bloco, sugestóes de inclusão, exclusão e realocação dos itens que os mesmos julgassem pertinentes.

\section{Segunda etapa}

O formulário de VD definido através do grupo focal foi apresentado para os coordenadores das Áreas Programáticas do município do Rio de Janeiro, para aprovação e indicação de farmacêuticos para aplicarem o instrumento. Os farmacêuticos que participaram receberam o formulário de visita domiciliar farmacêutica mais o manual de preenchimento da ferramenta. Posteriormente, eles tiveram três meses para aplicar a ferramenta nas visitas domiciliares. Após esse período, os profissionais responderam ao questionário de avaliação do instrumento.

O objetivo foi verificar se o formulário de VD estava adequado, isto é, se o instrumento estava adequado à rotina, apresentando informaçôes suficientes para a realização de visita domiciliar farmacêutica; claro, ou seja, se o instrumento facilitou a rotina, se é de fácil preenchimento e apresenta linguagem de fácil compreensão; efetivo/preciso, ou seja, se o instrumento auxilia como subsídio no processo de acompanhamento farmacoterapêutico auxiliando na identificação de problemas relacionados a medicamentos e no norteio da visita domiciliar farmacêutica; e exequível, se o instrumento está apropriado a ser utilizado pelos farmacêuticos na referida atividade. Ao final do questionário havia um campo aberto para sugestôes.

\section{Resultados e Discussão}

O grupo focal para a definição do formulário de visita domiciliar farmacêutica foi realizado com cinco participantes. Ressalta-se que, apesar do número de participantes ter sido apenas de cinco profissionais, observou-se saturação dos itens avaliados; desta forma foi possível a obtenção a construção da ferramenta. Os farmacêuticos participantes foram identificados como F01, F02, F03, F04 e F05.

O tempo de experiência destes profissionais na ESF variou de 1 a 4 anos, todos trabalhavam 40 horas semanais e três farmacêuticos trabalhavam em clínicas da família e os outros dois em centro municipal de saúde. 
A avaliação dos itens correspondentes ao "Perfil do paciente" teve índice de validade de conteúdo igual a 1 , pelos farmacêuticos. Nenhum dado foi excluído, mas alguns foram incluídos e outros realocados, pois segundo os participantes, poderá não ser possível a obtenção de alguns itens constantes nesse bloco antes da realização da visita. Como relatado pelos participantes:

[...] locais de armazenamento em casa e condições de moradia, como eu extraio isso do PE? Eu acho que você deveria colocar para a parte da visita [...] (F03).

A parte do cuidador também, porque nem sempre vai estar no PE. (F02).

Nem mobilidade muitas vezes. (F04).

Logo, "Locais de armazenamento dos medicamentos em casa", "Condiçóes de moradia", "Cuidador", "Com quem mora", "Escolaridade e Ocupação" e "Mobilidade" foram transferidos para a parte da obtenção dos dados durante a VD. É fundamental o conhecimento sobre as condiçôes de armazenamento dos medicamentos, para que, em casos de incoerências, as devidas orientaçóes sejam realizadas. Em inquéritos sobre farmácia domiciliar, foram encontrados de 3,8 a 5,1 medicamentos por domicílio. Boa parte desses medicamentos ainda é armazenada de forma inadequada, apresentando perda de qualidade e sendo descartada de forma prejudicial no meio ambiente (GASPARINI et al., 2011).

A "História Social" teve o índice de validade de conteúdo igual a 1. Portanto, nenhum dado foi excluído ou realocado. Porém no bloco "Farmacoterapia Atual" apesar de ter tido o IVC igual a 1, foram sugeridas mudanças. Este bloco apresenta informações que são obtidas na preparação e durante a VD. O adequado nível de conhecimento dos pacientes sobre a indicação dos medicamentos que utilizam é considerado fator essencial para a adesão ao tratamento farmacológico. Em seu estudo, concluíram que a maioria dos pacientes entrevistados possuía conhecimentos insuficientes para o uso seguro e eficaz dos medicamentos, o que sugere a necessidade de melhor orientação aos pacientes (MOREIRA et al., 2008) Diante disso, é necessário avaliar o conhecimento do paciente sobre sua farmacoterapia para que, caso necessário, sejam realizadas as orientaçôes farmacêuticas.

Em relação à pergunta "Para que você utiliza este medicamento?" ser realizada para cada medicamento utilizado pelo paciente, foi sugerida uma única pergunta para avaliação geral, conforme relatado: 
[...] poderia colocar uma pergunta só, geral: "Paciente consegue entender o objetivo da sua terapia", porque assim, eu acho que fica mais fácil do que você ir colocando item por item. (F05).

A pergunta que foi incluída no instrumento foi "Paciente consegue entender objetivo do tratamento medicamentoso?”. Serão pontuados os medicamentos que o paciente náo tiver entendimento do objetivo da terapia medicamentosa. Para avaliação da automedicação, um campo "observação" foi disponibilizado para ser preenchido caso o medicamento em uso não tenha sido prescrito.

As informaçôes sobre medicamentos mais demandadas pelos pacientes referem-se à posologia e indicação (LYRA JUNIOR; MARQUES, 2012). O desconhecimento dos nomes e dos regimes posológicos dos medicamentos prescritos pode causar erros graves no momento da utilizaçáo dos mesmos. Para o item "Posologia", as alterações sugeridas foram na descrição pelo paciente da "Posologia utilizada". Para otimizar o tempo e facilitar as anotações da informaçôes, foi sugerido a inclusão do termo "Posologia utilizada de acordo com a prescrita" colocando as opçóes Sim ( ) e Náo ( ).

Os farmacêuticos acharam pertinente acrescentar como os pacientes conseguem ter acesso aos medicamentos, com a inclusão do campo "Acesso ( ) Sim ( ) Não". A partir dessa informação podem-se realizar orientaçóes e direcionamentos que se fizerem necessários. Segundo F05:

Isso, pra cada item, porque aí você vai saber realmente quais os itens que ele está tendo dificuldade com acesso. (F05)

Segundo a OMS, cerca de um terço da população mundial não tem acesso a medicamentos essenciais, e $50 \%$ dos pacientes tomam de forma incorreta (OMS, 2012).

O IVC para o bloco "Problemas de Saúde/Queixas" foi igual a 1. Foram sugeridas algumas alteraçóes: mudança do campo aberto sobre os "Problemas de saúde" e a inclusão do campo "Queixas/Sintomas relatados pelos pacientes" com opçôes "fechadas", otimizando desta forma o preenchimento. Para o farmacêutico, o conhecimento das queixas e sintomas relatados pelos pacientes tem o objetivo principal de relacionar estes problemas aos medicamentos utilizados, a fim de conhecer a efetividade do tratamento, bem como aqueles problemas que podem ser causados ou agravados pelo uso, ou pela falta do uso. Sendo assim, é necessário avaliar as informaçôes contidas nesse bloco, pois podem ser indicativas de PRM. 
Os profissionais devem avaliar como o paciente compreende a doença e o tratamento, comunicar-lhes os benefícios do tratamento e discutir com eles as barreiras e os obstáculos que estejam influenciando na adesão (SOARES, 2016). Os profissionais acharam pertinente incluir uma área de observação da adesão, pois segundo eles, durante a conversa pode-se observar a falta de adesão devido a respostas contraditórias. Esse campo foi incluído no bloco "Farmacoterapia atual".

Eu tinha um paciente e eu sabia que ele tinha uma péssima adesão. Entáo eu vou perguntar isso pra ele e ele vai falar que sim, sim, sim, sim e eu sei que ele náo está tomando. Então a gente vai acabar avaliando. E quando você faz isso, a gente acaba tendo a visão se o paciente realmente está aderindo ou não. Aí você vai conseguir saber se a adesão está boa ou não fazendo só isso aqui. E no relato dele mesmo. (F05).

$\mathrm{Na}$ avaliação do bloco "Terapias alternativas/complementares", as terapias alternativas/complementares contêm uma ampla gama de abordagens terapêuticas: as ervas medicinais, os suplementos vitamínicos, as dietas especiais, a medicina chinesa, a homeopatia, as técnicas de relaxamento terapêutico entre outros Estudos de revisóes sistemática e meta-análise para avaliar os efeitos dessa terapia também estão aumentando (LEE et al., 2019). Aqui foi avaliada a importância das informaçóes sobre o conhecimento das terapias alternativas / complementares dos pacientes. Todos os farmacêuticos concordaram que não é necessário conter esse bloco de informaçóes. O IVC teve valor igual a 0. Sendo assim, este bloco foi excluído do formulário.

Todos os participantes concordaram que a identificação dos PRM é fundamental, tendo esse bloco o índice de validação de conteúdo igual a 1. Os participantes relataram:

Eu achei grande, mas não vi opção de diminuir (F04).

Eu coloquei extremamente importante e é tudo de marcar e você não perde muito tempo" (F05).

Alguns estudos de avaliação do serviço de atenção farmacêutica na otimização dos resultados terapêuticos, demonstraram que esta atividade facilita a identificação dos PRM (PLAKAS et al.,2016). Um PRM pode ser a causa da falha do tratamento e pode até desencadear novos problemas médicos, mais complexos que a doença tratada inicialmente (CALVO-SALAZAR et al., 2018). O propósito de identificar os problemas relacionados à farmacoterapia é ajudar os pacientes a atingirem suas metas terapêuticas e a obterem o máximo benefício do medicamento. equipe multidisciplinar permitem que os farmacêuticos desempenhem papel fundamental 
na melhoria dos resultados de saúde para os pacientes. Ao prestar o cuidado farmacêutico, o profissional se responsabiliza por garantir que o paciente possa cumprir os esquemas farmacoterapêuticos e seguir o plano assistencial, de forma a alcançar resultados positivos. A ênfase no cumprimento da dose, posologia, influência dos alimentos, interaçôes medicamentosas, o reconhecimento das potenciais RAM, entre outros, são elementos importantes no cuidado farmacêutico (PATEL et al., 2020) Cabe ressaltar que essa parte do formulário está na "análise e registros pósvisita". Logo deve ser preenchido de acordo com tudo que foi coletado e observado pelo farmacêutico durante a avaliação do paciente.

No bloco "Finalização, agendamento e outras ações pactuadas com o paciente", o índice de validação de conteúdo foi 0 . Segundo a análise de discurso do grupo focal, todos os farmacêuticos concordaram que não é viável e importante ter esse bloco no formulário. No entanto, os participantes sugeriram a inclusão dos itens "Hora de início da visita" e "Hora de término da visita" como sendo um dado importante e passível de comparaçóes futuras. Sendo assim, foi incluído no do bloco referente à realização da visita a hora de início e a hora de término da visita domiciliar farmacêutica. As principais mudanças ocorridas entre a versão 1 (pesquisa bibliográfica), 2 (grupo focal) e 3 (teste piloto) estão descritas no quadro 1.

Quadro 1. Mudanças realizadas no formulário desenvolvido com base na literatura após o grupo focal e o teste piloto com farmacêuticos atuantes na Estratégia da Saúde da Família do município do Rio de Janeiro. Rio de Janeiro, 2020

\begin{tabular}{|l|l|}
\hline Bloco do formulário & Alteraçóes propostas e aceitas \\
\hline Perfil do paciente & $\begin{array}{l}\text { Campo realocado (para bloco história social): Locais de armazenamento } \\
\text { dos medicamentos em casa; Condiçóes de moradia; Cuidador; Com quem } \\
\text { mora; Escolaridade e Ocupação; Mobilidade. } \\
\text { Campo incluído: Comorbidades; Exames laboratoriais }\end{array}$ \\
\hline História social & Náo houve modificação \\
\hline Farmacoterapia atual & $\begin{array}{l}\text { Campo excluído: Terapias alternativas/complementares. } \\
\text { Campos Incluídos: Acesso ao medicamento; observaçôes e Exames } \\
\text { Complementares". }\end{array}$ \\
\hline $\begin{array}{l}\text { Acesso aos } \\
\text { medicamentos }\end{array}$ & $\begin{array}{l}\text { Bloco excluído, a informação de acesso aos medicamentos foi incluída no } \\
\text { bloco da farmacoterapia atual. }\end{array}$ \\
\hline
\end{tabular}




\begin{tabular}{|l|l|}
\hline Bloco do formulário & Alteraçóes propostas e aceitas \\
\hline $\begin{array}{l}\text { Problemas de saúde/ } \\
\text { queixas }\end{array}$ & $\begin{array}{l}\text { Campos incluídos: Sintomas e PRM. Foram realizadas alteração do } \\
\text { campo aberto sobre os "Problemas de saúde" e a inclusão do campo } \\
\text { "Queixas/Sintomas relatados pelos pacientes" com opções "fechadas" } \\
\text { otimizando, assim, o preenchimento. }\end{array}$ \\
\hline $\begin{array}{l}\text { Terapias alternativas/ } \\
\text { complementares }\end{array}$ & Bloco excluído \\
\hline $\begin{array}{l}\text { Problemas relacionados } \\
\text { a medicamentos }\end{array}$ & Não houve modificação \\
\hline $\begin{array}{l}\text { Intervençóes } \\
\text { farmacêuticas }\end{array}$ & Não houve modificação \\
\hline $\begin{array}{l}\text { "Finalização, } \\
\text { agendamento e outras } \\
\text { ações pactuadas com o } \\
\text { paciente" }\end{array}$ & Bloco excluído \\
\hline
\end{tabular}

Fonte: elaboração dos autores.

Na ESF do município do Rio de Janeiro, os profissionais de saúde que atuam na visita domiciliar realizam reuniôes periódicas para avaliação das necessidades dos pacientes e o planejamento do plano de ação. A proposta é que todas as informaçóes obtidas durante a visita domiciliar farmacêutica, bem como as conclusôes deste processo, devam ser discutidas nessas reunióes, de forma a contribuir para o melhor atendimento ao paciente, visando à integralidade da atençáo à saúde.

A segunda etapa do trabalho consistiu na aplicação da ferramenta de visita domiciliar farmacêutica. Para isso foram convidadas a participar as dez Áreas Programáticas do município, das quais sete aceitaram participar da pesquisa. Os farmacêuticos foram indicados pelos coordenadores de cada Área Programática a participarem nesta fase, totalizando 32 farmacêuticos que efetivamente realizavam visita domiciliar farmacêutica. Os farmacêuticos que participaram do grupo focal, primeira etapa, náo participaram da segunda etapa.

Para a avaliação do item "Adequação", todos participantes da pesquisa, 100\% (32), consideraram que o instrumento está adequado para a rotina de trabalho, que compreende a obtenção de informaçôes suficientes para o desenvolvimento da visita domiciliar farmacêutica. Como uma das atividades intrínsecas às equipes de ESF, a visita domiciliar proporciona ao profissional adentrar o espaço da família e, assim, 
identificar suas demandas e potencialidades (PATEL et al., 2020) É o momento adequado para levar até os pacientes, que por algum motivo não conseguem ter acesso à unidade de saúde, as açôes que acontecem na unidade (CREPALDI, 2009).

Para a avaliação do item "Clareza", todos os participantes, 100\% (32), concordaram que o instrumento é de fácil preenchimento e apresenta linguagem de fácil compreensão. $\mathrm{O}$ instrumento também serviu como subsídio no processo de acompanhamento farmacoterapêutico contemplando as análises e registro pós-visita nas últimas etapas.

Apesar da existência de formulários para o atendimento farmacêutico, como por exemplo, o Método DÁDER, PWDT (Pharmacist's Work up of Drug Therapy) e TOM (Therapeutic Outcomes Monitoring), é necessário um formulário próprio e adaptado para registro das atividades realizadas e pactuadas com o paciente e a família no atendimento farmacêutico domicilia (SOARES, 2016).

O PWDT e o Dáder possuem documentos mais estruturados, porém mais complexos, para o atendimento ao paciente, levando ao aumento do tempo de consultas (BEZERRA et al., 2015). Isso pode ser fator limitante quando se realiza VD com atuação multiprofissional. Na maior parte das visitas, o farmacêutico vai junto com outros profissionais de saúde, e muitas vezes são feitas mais de uma visita no mesmo período do dia para aproveitar o deslocamento. Desta forma, ferramentas complexas podem acabar dificultando a atuação do profissional. $\mathrm{O}$ uso de um instrumento que facilite o desenvolvimento de um processo passo a passo, com vistas a aperfeiçoar o plano farmacoterapêutico, tem-se mostrado benéfico (GALASSI et al., 2014).

No acompanhamento farmacoterapêutico, o profissional se responsabiliza por garantir que o paciente possa cumprir os esquemas farmacoterapêuticos e seguir o plano assistencial, de forma a alcançar resultados positivos. A ênfase no cumprimento da dose, posologia, influência dos alimentos, interaçóes medicamentosas, reconhecimento de reaçóes adversas a medicamentos potenciais, entre outros, são elementos importantes no cuidado farmacêutico (NAKAMURA; LEITE, 2016).

Para a avaliação do item "Efetividade/Precisão", todos os farmacêuticos participantes, $100 \%$, apontaram que o formulário é efetivo e preciso na identificação e resolução dos problemas relacionados a medicamentos. O propósito de identificar os problemas relacionados à farmacoterapia é ajudar os 
pacientes a atingirem suas metas terapêuticas e obterem o máximo de benefício do medicamento (FERREIRA; MELO, 2016).

O formulário em avaliação é voltado para nortear e facilitar o atendimento da visita domiciliar farmacêutica na rotina desses profissionais. Atualmente nesse processo de trabalho, tais demandas são geralmente heterogêneas e variam entre as unidades de saúde, que, por sua vez, atendem de forma abrangente às diferentes necessidades. A incorporação de farmacêuticos em equipes multidisciplinares de atendimento ambulatorial é benéfica e deve ser fortemente considerada pelos clínicos e formuladores de políticas de saúde. Neste contexto, há evidências das intervenções farmacêuticas na prevenção e na solução dos PRM, otimizando o uso de medicamentos e melhorando os resultados em saúde (ABDULRHIM et al., 2020), o que enfatiza a necessidade de formulários bem estruturados para o atendimento domiciliar farmacêutico.

Para a avaliação dos itens referentes à Adequação, Clareza e Efetividade/ Precisão, todos os participantes concordaram que o instrumento pode ser utilizado pelos farmacêuticos sem alteraçôes. Porém, quanto à avaliação do item referente a Exequibilidade, 18,7\% (06) questionaram a falta de um campo para preenchimento dos exames complementares. Essa observação foi atendida e incluída no formulário. Os exames complementares devem ser solicitados como forma de direcionar e ajudar no diagnóstico da doença, podendo incluir os de caráter laboratorial e de imagem (HEPLER; STRAND, 1990).

Os parâmetros de exames diagnósticos estão inseridos nas políticas públicas de saúde. Esta assume papel importante na prevenção, diagnóstico, tratamento, acompanhamento das enfermidades em geral e são preponderantes na qualidade de vida do paciente A necessidade do conhecimento por parte do farmacêutico é explicada pelo fato de o sucesso terapêutico obtido para cada tratamento em uso pelo paciente ser analisado pelo estado clínico atual dos problemas em saúde. A verificação dos parâmetros clínicos, laboratoriais e complementares mostra se o objetivo terapêutico foi ou não alcançado. Os dados para adequação, clareza, efetividade / precisão, uma exequibilidade e sugestôes da ferramenta estão descritas no quadro 2. 
Quadro 2. Avaliação do Instrumento de Visita Domiciliar Farmacêutica na Estratégia de Saúde da Família do município do Rio de Janeiro. Rio de Janeiro, 2020

\begin{tabular}{|c|c|c|c|c|}
\hline Dados & Perguntas & $\begin{array}{l}\text { Farmacêuticos } \\
\text { (n) }\end{array}$ & $\begin{array}{c}\text { Porcentagem } \\
\text { (\%) }\end{array}$ & Sugestóes \\
\hline Adequação & $\begin{array}{l}\text { a) O instrumento está adequado na } \\
\text { sua rotina? } \\
\text { b) O instrumento apresenta } \\
\text { informaçốes suficientes para } \\
\text { realizaçáo de visita domiciliar } \\
\text { farmacêutica? } \\
\text { c) O Instrumento facilitou sua } \\
\text { rotina? }\end{array}$ & 32 & 100 & Sem sugestóes \\
\hline Clareza & $\begin{array}{l}\text { a) O instrumento é de fácil } \\
\text { preenchimento? } \\
\text { b) O instrumento apresenta } \\
\text { linguagem de fácil compreensão? } \\
\text { c) O instrumento auxiliou } \\
\text { como subsídio no processo } \\
\text { de acompanhamento } \\
\text { farmacoterapêutico? }\end{array}$ & 32 & 100 & Sem sugestóes \\
\hline $\begin{array}{l}\text { Efetividade/ } \\
\text { Precisão }\end{array}$ & $\begin{array}{l}\text { a) O instrumento auxiliou na } \\
\text { identificação de problemas } \\
\text { relacionados a medicamentos? } \\
\text { b) O instrumento auxiliou a nortear } \\
\text { a visita domiciliar farmacêutica? }\end{array}$ & 32 & 100 & Sem sugestóes \\
\hline Exequibilidade & $\begin{array}{l}\text { O instrumento está apropriado a } \\
\text { ser utilizado pelos farmacêuticos } \\
\text { na ESF? }\end{array}$ & 26 & 86 & $\begin{array}{l}\text { Incluir campo: } \\
\text { Exames } \\
\text { complementares }\end{array}$ \\
\hline
\end{tabular}

Fonte: elaboração dos autores.

\section{Considerações finais}

Foi possível desenhar e avaliar o instrumento de visita domiciliar farmacêutica na realização do cuidado farmacêutico domiciliar na ESF do município do Rio de Janeiro. A avaliação do novo instrumento foi considerada positiva, pois foi realizada através de uma amostra representativa da população de farmacêuticos que realizam visita domiciliar na ESF do referido município.

Pode-se concluir que o instrumento é viável para ser utilizado, necessitando apenas de acréscimo do campo referente a exames complementares, a fim de atender às sugestôes dos participantes. ${ }^{1}$ 


\section{Referências}

ABDULRHIM, S. et al. The impact of pharmacist care on diabetes outcomes in primary care settings: An umbrella review of published systematic reviews. Prim Care Diabetes, v. S17519918, n. 19, p. 30425-5, jan. 2020.

AIZENSTEIN, M. L.; TOMASSI, M. H. Problemas relacionados a medicamentos; reaçóes adversas a medicamentos e erros de medicação: a necessidade de uma padronização nas definições e classificações. Rev Ciênc Farm Básica Apl., v. 32, n. 2, p. 169-173, 2011.

ALMEIDA, M. C. P.; MISHIMA, S. M. O. desafio do trabalho em equipe na atenção à Saúde da Família: construindo "novas autonomias" no trabalho. Interface. Comunicação, Saúde, Educação. p. 150-153, 2001.

BEZERRA, M. I. C.; LIMA, M. J.; LIMA, Y. C. A visita domiciliar como ferramenta de cuidado da fisioterapia na estratégia saúde da família. $S A N A R E$. Sobral, v. 14, n. 1, p. $7680,2015$.

BORGES, C. D.; SANTOS, M. A. Aplicaçóes da técnica do grupo focal: fundamentos metodológicos, potencialidades e limites. Sociedade de Psicoterapias Analíticas Grupais do Estado de São Paulo, v. 6, n. 1, p. 74-80, 2005.

BORGES, R.; D’OLIVEIRA, A. F. P. L. A visita médica como espaço para interação e comunicação em Florianópolis, SC. Interface - Comunic., Saude, Educ., v. 15, n. 37, p. 46172, 2011.

BRASIL. Conselho Federal de Farmácia. RE no 386/2002. Dispóe sobre as atribuiçóes do farmacêutico no âmbito da assistência domiciliar em equipes multidisciplinares. Acesso em: 20 out. 2017. Disponível em http://www.cff.org.br/userfiles/file/resolucoes/386.pdf.

Ministério da Saúde. Portaria no 963, de 27 de maio de 2013. Redefine a Atenção Domiciliar no âmbito do Sistema Único de Saúde (SUS). Diário Oficial da União, 2013. Acesso em: 5 mar. 2017. Disponível em: http://bvsms.saude.gov.br/bvs/saudelegis/gm/2013/ prt0963_27_05_2013.html>.

. Ministério da Saúde. Secretaria de Atenção à Saúde. Departamento de Atenção Básica. Programa Saúde da Família: ampliando a cobertura para consolidar a mudança do modelo de Atenção Básica. Rev. bras. saúde matern. infant. Brasília, v 3, n. 1, p. 113-125, 2003.

- Ministério da Saúde. Secretaria de Atenção à Saúde. Departamento de Atenção Básica. Caderno de atenção domiciliar. Brasília: Ministério da Saúde, 2013.

. Ministério da Saúde. Secretaria de Atenção à Saúde. Departamento de Atenção Básica. Política Nacional de Atenção Básica. Brasília: Ministério da Saúde, 2012. 
- Ministério da Saúde. Secretaria de Ciência, Tecnologia e Insumos Estratégicos. Departamento de Assistência Farmacêutica e Insumos Estratégicos. Cuidado farmacêutico na atenção básica. - 1. ed. rev. - Brasília: Ministério da Saúde, 2015. Disponível em <http:// bvsms.saude.gov.br/bvs/publicacoes/servicos_farmaceuticos_atencao_basica_saude_1 ed.pdf>. Acesso em: 15 mar 2017.

- Ministério da Saúde. Secretaria de Ciência, Tecnologia e Insumos Estratégicos. Departamento de Assistência Farmacêutica Insumos Estratégicos. Planejamento e implantação de serviços de cuidado farmacêutico na Atenção Básica à Saúde: a experiência de Curitiba. Brasília : Ministério da Saúde, 2014.

CALVO-SALAZAR, R. A. et al. Problemas relacionados con medicamentos que causan ingresos por urgencias en un hospital de alta complejidad. Farm Hosp., v. 42, n. 6, p. 228-233, nov 2018.

CARDOSO, C. K. et al. Atenção farmacêutica domiciliar: série de casos de usuários do programa práticas integradas em saúde coletiva. Rev Ciênc Farm Básica Apl., v. 34, n. 2, p. 263268, 2013.

CENTA, M. L.; ALMEIDA, B. M. O Programa de Saúde da Família sob o olhar da Equipe Multidisciplinar. Fam. Saúde Desenv. Curitiba, v. 5, n. 2, p. 103-113, 2003.

CORRER, C. J.; OTUKI, M. F.; SOLER, O. Assistência farmacêutica integrada ao processo de cuidado em saúde: gestão clínica do medicamento. Revista Pan-Amazônica de Saúde, v. 2, n. 3, p. 41-49, 2011.

FERREIRA, V. L.; MELO, M. L. A importância do seguimento farmacoterapêutico na saúde: uma revisão da literatura. Visão Acadêmica Curitiba, v. 17, n. 1, p. 125-136, 2016.

FIGUEIREDO, E. N. A Estratégia Saúde da Família na Atenção Básica do SUS, UNA-SUS, UNIFESP, 2015. São Paulo, 2015. Available from: http://www.unasus.unifesp.br/biblioteca virtual/esf/2/unidades_conteudos/unidade05. Acesso em: 23 out. 2017.

GALASSI, C. V. et al. Atenção domiciliar na atenção primária à saúde: uma síntese operacional. ABCS Health Science, v. 39, n. 3, p. 177-185, 2014.

GASPARINI, J. C.; GASPARINI, A. R.; FRIGIERI, M. C. Estudo do descarte de medicamentos e consciência ambiental no município de Catanduva/ SP. Ciência \& Tecnologia, v. 2, n. 1, p. 38-51, 2011.

HEPLER, C. D.; STRAND, L. M. Opportunities and responsibilities in pharmaceutical care. Am J Hosp Pharm, v. 47, n. 3, p. 533-43, 1990.

KIND, L. Notas para o trabalho com a técnica de grupos focais. Psicologia em Revista, Belo Horizonte, v. 15, n. 10, p. 124-136, 2004. 
LEE, S. M.; CHOI, H. C.; HYUN, M. K. An Overview of Systematic Reviews: Complementary Therapies for Cancer Patients. Integr Cancer Ther., v. 18, p. $1534735419890029,2019$.

LYRA JUNIOR, D. P.; MARQUES, TC. As bases da dispensação racional de medicamentos para farmacêuticos. 2 ed. Pharmabooks. São Paulo, 2012.

MOREIRA, L. B. M. et al. Conhecimento sobre o tratamento farmacológico em pacientes com doença renal crônica. Brazilian Journal of Pharmaceutical Sciences, v. 44, n. 2, 2008.

NAKAMURA, C. A.; LEITE, S. N. A construção do processo de trabalho no Núcleo de Apoio à Saúde da Família: a experiência dos farmacêuticos em um município do sul do Brasil. Ciência \& Saúde Coletiva, n. 5, v. 21, p. 1565-1572, 2016.

OLIVEIRA, E. M.; SPIRI, W. C. Programa Saúde da Família: a experiência de equipe multiprofissional. Rev Saúde Pública, v. 40, n.4, p. 727-733, 2006.

OLIVEIRA, L. C.; ASSIS, M. M.; BARBONI, A. R. Assistência Farmacêutica no Sistema Único de Saúde: da Política Nacional de Medicamentos à Atenção Básica à Saúde. Ciência \& Saúde Coletiva, v. 15, supl. 3, p. 3561-3567, 2010.

ORGANIZAÇÃO MUNDIAL DA SAÚDE. Servicios farmacéuticos basados en la atención primaria de salud. Documento de posición de la OPS/OMS. Washington: OPS; 2002 (La Renovación de la Atención Primaria de Salud en las Américas).

PATEL, B. K. et al. Integrar farmacêuticos em equipes de atendimento: um protocolo de revisão sistemática qualitativa. JBI System Database Rev Implementar Rep., jan. 2020.

PEREIRA, N. C.; LUIZA, V. L.; CRUZ, M. M. Serviços farmacêuticos na atenção primária no município do Rio de Janeiro: um estudo de viabilidade. Saúde e Debate, v. 39, n. 105, p.451468, 2015.

PLAKAS, S. et al. Validation of the 8-Item Morisky Medication Adherence Scale in Chronically Ill Ambulatory Patients in Rural Greece. Open Journal of Nursing, v. 6, p. 158169, 2016.

RESSEL, L. B. et al. O uso do grupo focal em pesquisa qualitativa. Texto Contexto Enferm. Florianópolis, v. 17, n. 4, p. 779-86, 2008.

SAVASSI, L. C.; DIAS, M. F. Grupos de estudo em Saúde da Família: módulo visita domiciliar. 2006. Disponível em: http://www.smmfc.org.br/gesf/gesf_vd.htm>. Acesso em: 4 out. 2017.

SILVA, J. R.; ASSIS, S. M. Grupo focal e análise de conteúdo como estratégia metodológica clínica-qualitativa em pesquisas nos distúrbios do desenvolvimento. Cadernos de pós-graduação em distúrbios, São Paulo. v. 10, n. 1, p. 146-152, 2010.

SILVA, R. M. et al. Assistência farmacêutica no município do Rio de Janeiro, Brasil: evolução de aspectos selecionados de 2008 a 2014. Ciência \& Saúde Coletiva, v. 21, n. 5, p. 1421-1432, 2016. 
SOARES, E. V. B. Atenção Básica e Informação: análise do Sistema de Informação em Saúde para Atenção Básica (SISAB) e estratégia e-SUS AB e suas repercussöes para uma gestão da saúde com transparência. Universidade de Brasília: Faculdade de Economia, Administração e Contabilidade. Brasília, 2016. p. 1-42.

\section{Nota}

${ }^{1}$ J. B. dos Santos e T. M. Luquetti: elaboração do projeto de pesquisa, realização da coleta, análise e interpretação dos dados e redação do artigo. S. R. Castilho e S. C. Elias: supervisão da elaboração do projeto, contribuição para a análise e interpretaçáo dos dados, redação e revisão crítica do artigo. 


\section{Abstract}

\section{Home Pharmaceutical Care in the Family Health Strategy}

This work developed and evaluated a tool to assist in the planning, execution, data recording and evaluation of the actions of the pharmaceutical home visit in the Family Health Strategy team. The first phase comprised the construction of the pharmaceutical home visit tool, through a focus group and discourse analysis. The professionals' view on the role of the pharmacist and his professional practice was discussed, especially in home visits in primary health care units. In the second phase, the form developed in the previous step was used during the home visit by 32 pharmacists, and adequacy, clarity, effectiveness / precision, feasibility and suggestions for the tool were evaluated. All study participants effectively perform home visits in the Family Health Strategy in the city of Rio de Janeiro. According to an expressive evaluation of the participants, the instrument was considered adequate, clear, effective, precise and feasible for use in the practice of home visits, and is an important assistance to the professional in performing home pharmaceutical care in that city.

Keywords: Primary Health Care; pharmaceutical care; home visit. 